\title{
Approvisionnement En Eau Dans La Ville De Brazzaville, Congo
}

\section{Nkounkou Loumpangou Célestine (Docteur, Maître-Assistant) Louzayadio Mvouezolo Raison Félicien (Doctorant)}

Unit of Chemistry of Vegetal and Life, Faculty of Sciences and Techniques,

Marien N'gouabi University, B.P. 69, Brazzaville, Congo

Ayessou Nicolas (Docteur, Maître des Conférences)

Laboratory of Analysis and Testing, Ecole Supérieure Polytechnique,

University Cheikh Anta Diop, BP.5085, Dakar

Elouma Ndinga Arnold Murphy (Docteur, Assistant)

Ngakegni-Limbili Adolphe Christian (Docteur, Assistant)

Unit of Chemistry of Vegetal and Life, Faculty of Sciences and Techniques,

Marien N'gouabi University, B.P. 69, Brazzaville, Congo

Mar Diop Codou Gueye (Professeur Titulaire)

Laboratory of Analysis and Testing, Ecole Supérieure Polytechnique,

University Cheikh Anta Diop, BP.5085, Dakar

Ouamba Jean- Maurille (Professeur Titulaire)

Unit of Chemistry of Vegetal and Life, Faculty of Sciences and Techniques,

Marien N'gouabi University, B.P. 69, Brazzaville, Congo

doi: 10.19044/esj.2017.v13n21p474 URL:http://dx.doi.org/10.19044/esj.2017.v13n21p474

\section{Abstract}

The difficulty of water supply is a major problem faced by the households of Brazzaville in Congo. The objective of this study is to list and identify the different types of water used, and to assess the general hygiene of households. The method used in this prospective study is based on an onsite survey and direct measurement. The survey involves administering a questionnaire to the households in the 9 districts of the city of Brazzaville. 630 households were surveyed. 70 households were drawn in this study and were selected in each borough. There are many sources of water. Overall, $80.16 \%$ of households consume water from the SNDE. The survey revealed that $71.00 \%$ of households walk on foot to get access to water supply. Plastic cans and buckets are used more in the transportation (43.00 and $37.00 \%$ ) and storage (45.00 and $39.00 \%)$ of water. The water storage period is relatively long. It can hold water from 3 days $(21.00 \%)$ to more than one week $(13.00 \%)$ for some households. Analysis of the results of the survey 
showed that the sources of water supply are diversified. This diversification is due to the phenomenon of shortage and/or repeated water cut recorded by households. Water supply, transport and storage practices, and the cleaning of water containers highlight the precarious conditions of hygiene and sanitation households in the city of Brazzaville.

Keywords: Survey, supply, drinking water, hygiene, Brazzaville.

\section{Résumé}

Les difficultés d'approvisionnement en eau sont des problèmes réels et majeurs que vivent les ménages de Brazzaville. L'objectif de cette étude est de répertorier, d'identifier les différents types d'eaux utilisées et d'évaluer l'hygiène générale des ménages. La méthode utilisée dans cette étude prospective est basée sur une enquête par interview sur place et mesure directe. L'enquête a consisté à administrer aux ménages un questionnaire dans les 9 arrondissements de la ville de Brazzaville. 630 ménages ont été enquêtés en raison de 70 ménages tirés puis sélectionnés dans chaque arrondissement. Les sources d'approvisionnement en eaux enregistrées sont multiples. Dans 1'ensemble, 80,16\% des ménages consomment l'eau de la SNDE. L'enquête a révélé, que $71,00 \%$ des ménages se déplacent à pied pour l'approvisionnement en eau. Les bidons et les seaux en plastiques sont plus utilisés dans le transport $(43,00$ et $37,00 \%)$ et pour le stockage $(45,00$ et $39,00 \%$ ) de l'eau. La durée de stockage de l'eau est relativement longue, de 3 jours $(21,00 \%)$ à plus d'une semaine $(13,00 \%)$ pour certains ménages. L'analyse des résultats de l'enquête a montré que, les sources d'approvisionnement en eau sont diversifiées. Cette diversification est due au phénomène de pénurie et/ou coupure d'eau répétée enregistrées par les ménages. Les pratiques d'approvisionnement, de transport et de stockage en eau ; mais aussi le nettoyage des récipients mettent en évidence la précarité des conditions d'hygiène et d'assainissement des ménages de la ville Brazzaville.

Mots-clés: Enquête, approvisionnement, eau de consommation, hygiène, Brazzaville.

\section{Introduction}

La forte croissance démographique des pays en voie de développement pose des enjeux spatiaux qui se traduisent surtout par un fort taux d'urbanisation. Au Congo, et à Brazzaville en particulier, l'indice de concentration urbaine est passé de $46 \%$ en 1960 à $65 \%$ en 2015 (Koua, et al., 2011). Cette croissance urbaine ne s'accompagne pas de mesures en 
adéquation avec les besoins de la population sur l'approvisionnement en eau, l'hygiène et l'assainissement de l'environnement.

Par ailleurs, le Congo dispose chaque année en moyenne 222 milliards de $\mathrm{m}^{3}$ d'eau de surface, 198 milliards de $\mathrm{m}^{3}$ d'eau souterraine renouvelable, auxquels il faut ajouter plus de 1000 milliards de $\mathrm{m}^{3}$ d'eau venant des pays voisins (Makouési, et al., 2011). Brazzaville, capitale politique du pays, dispose d'énormes potentielles hydriques notamment le fleuve Congo, le Djoué, la rivière Djiri, ... La production et la distribution d'eau potable sont essentiellement assurées depuis 1967 par la Société Nationale de Distribution d'Eau (SNDE). Ce réseau de distribution d'eau potable souffre essentiellement de l'ancienneté de ses installations et de la dégradation des équipements connexes. Le taux de desserte et de raccordement en eau est respectivement 78 et $36 \%$ dans la ville de Brazzaville. Malgré ce potentiel hydrique dont dispose la ville de Brazzaville et un taux de desserte élevé, les populations sont obligées de s'approvisionner de manière informelle (branchements privés, porteurs d'eau, sources naturelles, eau de forage et de puits). Ainsi, de sérieuses perturbations et des ruptures dans la distribution d'eau potable se produisent dans certains quartiers de Brazzaville. Il ne passe pas des semaines sans qu'il ait des coupures d'eau. Des quartiers entiers sont privés de l'eau depuis des mois, voire des années (Batantou, 2013).

Ainsi se développent de nombreuses maladies d'origine hydrique comme le choléra, la fièvre typhoïde, les infections gastro-intestinales, ...

L'analyse de la situation d'eau potable met en évidence la précarité des conditions d'approvisionnement en eau aussi bien en milieu urbain que rural. Par conséquent, cette étude se propose de répertorier, d'identifier les différents types d'eaux utilisées et d'évaluer l'hygiène générale des ménages dans la ville de Brazzaville.

\section{Méthodologie d'enquête}

\section{Zone d'étude}

La zone urbaine de Brazzaville d'une superficie de $263,9 \mathrm{~km}^{2}$, est située sur la rive droite du fleuve Congo. Administrativement, Brazzaville est découpée en neufs (09) arrondissements à savoir : Makélékélé (1), Bacongo (2), Poto-poto (3), Moungali (4), Ouenzé (5), Talangaï (6), M'filou (7), Madibou (8) et Djiri (9). Située dans le département du pool, cette ville est fortement peuplée soit 1.606.529 habitants en 2015 avec une densité de 5.204 hab. $/ \mathrm{km}^{2}$ (Koua, et al., 2011) (Figure 1). Soixante-dix (70) ménages par arrondissement ont été tirés puis sélectionnés, soit un total de 630 ménages enquêtés. 


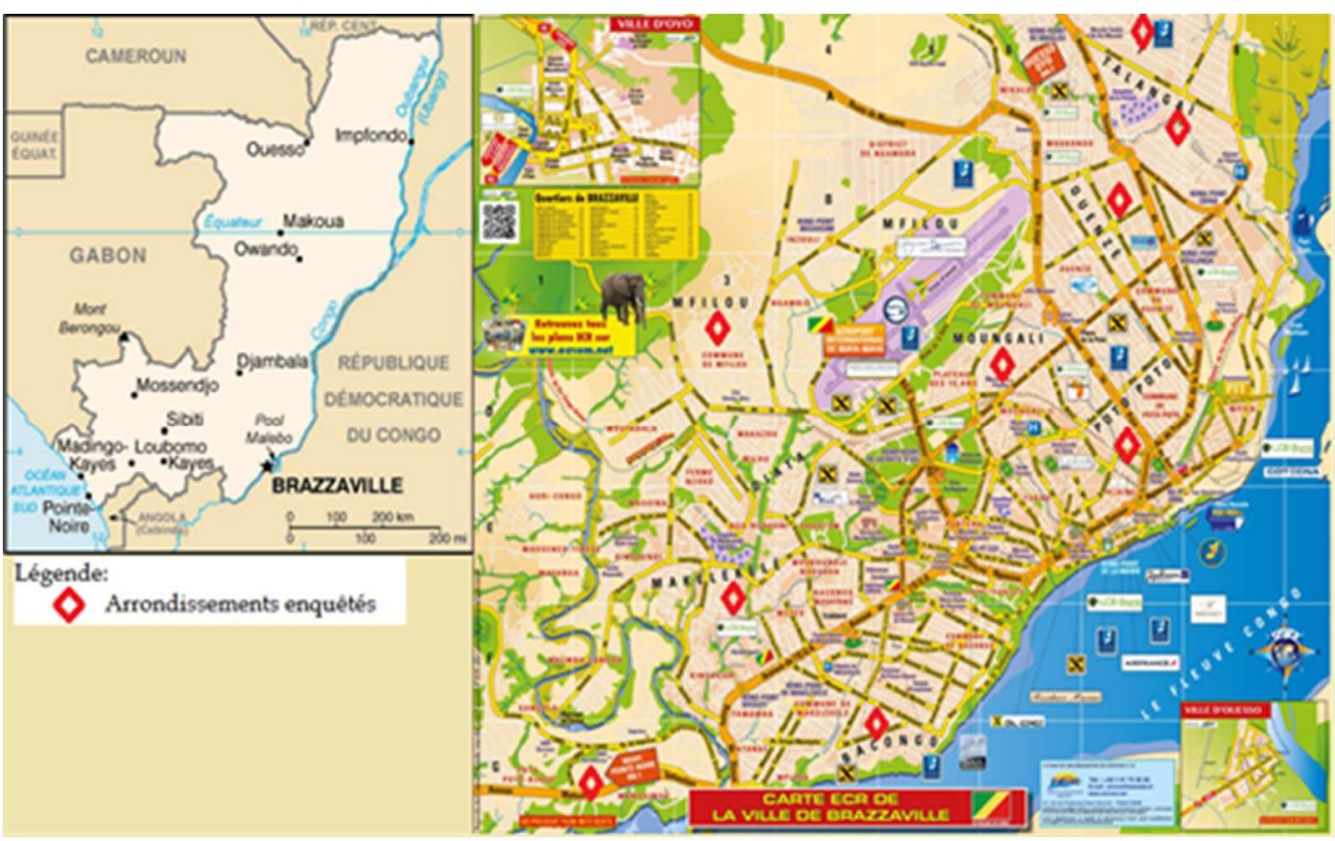

Figure 1. Carte de la ville Brazzaville-Congo

Source : Editions et communications Régionales (ECR), 2017

\section{Pré-enquête}

Cent (100) ménages ont été tirés, puis sélectionnés dans quatre arrondissements (Makélékélé, Bacongo, Moungali et M'filou) de la ville de Brazzaville (Figure 1). La pré-enquête a été effectuée dans le but de repérer les erreurs de présentation de la fiche, identifier les problèmes de codification des variables et modalités lors du dépouillement des fiches sur le logiciel utilisé et suggérer les catégories de réponses supplémentaires qui peuvent être codées d'avance dans le questionnaire (Statistique Canada, 2003).

\section{Enquête}

Après la pré-enquête, une enquête proprement dite a été réalisée dans les neuf arrondissements que compte la ville de Brazzaville.

Pour être le plus représentatif possible, la méthode de sondage utilisée est l'échantillonnage commun à plusieurs degrés. Elle a été constituée de l'échantillonnage par grappes à trois degrés à l'aide d'une base géographique au premier degré pour sélectionner les neuf arrondissements de la ville de Brazzaville (unités primaires d'échantillonnage); au deuxième degré, des zones de dénombrement (unités secondaires d'échantillonnage) de manière à prendre en compte, les diversités susceptibles d'être constatées au sein des arrondissements et enfin, d'un échantillon systématique de ménages (unités tertiaires d'échantillonnage) dans une parcelle, au troisième degré (OMS, 2010; Statistique Canada, 2003). 
Explicitement, chaque arrondissement a été subdivisé en zone de dénombrement. Le tirage prend en compte les listes des zones de dénombrement et les blocs disponibles de façon à couvrir la zone géographique de chaque arrondissement. Des pas de sondage variant d'une zone de dénombrement à l'autre ont été appliqués pour la sélection des parcelles. Cependant, la table des nombres aléatoires a été utilisée pour le choix de ménage à interviewer.

Pour la fiabilité des informations et des réponses aux questions posées, les personnes ayant 18 ans et plus ont été éligibles et interrogées.

Sur le terrain, un arrondissement a été enquêté par semaine ; soit une capacité journalière de 10 ménages pour tenir compte des difficultés de transport et de la charge de travail. En cas de collecte insuffisante des données, la méthode de proximité géographique a été appliquée, pour compléter ou reprendre le recueil d'informations. Les problèmes d'accessibilité et refus ont été notés. Un ménage ayant refusé l'interview, a été remplacée aussitôt par un autre, dans le sens de progression du pas de sondage.

Les interviews ont été réalisées en langue française et les deux langues nationales (Lingala et Kituba), selon les arrondissements et le niveau social de la population. Le point de départ de l'enquête dans chaque arrondissement a été choisi volontairement.

\section{Traitement et analyse des données}

Le dépouillement des fiches d'enquête a été accompli à l'aide du logiciel CSPro 5.0 développé par le Bureau de Recensement des Etats-Unis. La vérification de chaque fiche d'enquête a été effectuée minutieusement dans le fichier de dépouillement. Après dépouillement, la base des données obtenues a été ensuite transportée dans le logiciel SPSS Statistics 17.0 pour la production des tableaux bruts. Ces tableaux ont été à leurs tours transportés dans Excel 2007 pour produire les courbes, histogrammes et le test de chi-deux.

\section{Résultats et discussion}

\section{Caractéristiques sociodémographiques}

\subsection{Groupe d'âge par arrondissement}

La Figure 2 présente l'effectif des ménages enquêtés par tranche d'âge par arrondissement (soit 70 ménages). D'une manière générale, 14,60\% ; $36,35 \% ; 23,65 \% ; 13,49 \%$ et $11,90 \%$ des personnes ont été enquêtées pour des tranches d'âge respectives d'au plus 20 ans, 21 - 30 ans, 31- 40 ans, $40-50$ ans et d'au moins 51 ans. Nous observons que, la tranche d'âge (de 
21 - 30 ans) est plus représentée avec un taux de $36,35 \%$, soit un effectif de 17 à 30 ménages par arrondissements. Par contre, les personnes du troisième âge représentent un faible effectif (4 à 15 personnes) dans les neuf arrondissements respectifs, soit un taux de 11,90\% enquêtés. Ces résultats montrent que, les personnes interviewées dans cette étude sont majoritairement jeunes.

Figure 2. Répartition des enquêtés par tranches d'âge par arrondissement P-value $=0,00000 ; X^{2}=46,19426$

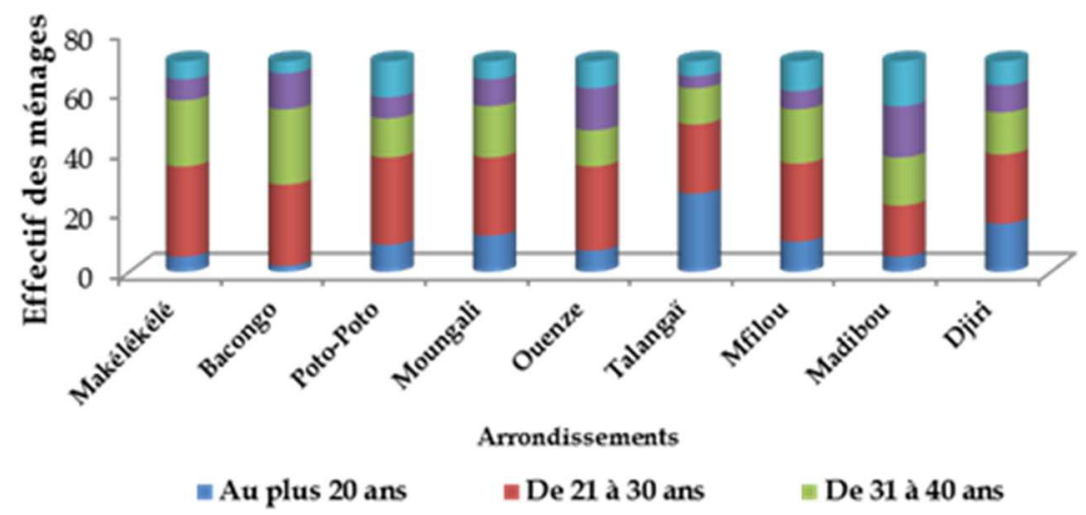

\subsection{Sexe des répondants par arrondissement}

Sur un total de 630 personnes enquêtées, les femmes représentent 54,92 $\%$ contre 45,08 \% des hommes. Cependant, dans les arrondissements Talangaï et Djiri, le taux des hommes est moyennement élevé $(52,86 \%$ et $51,43 \%$ ) contre $47,14 \%$ et $48,57 \%$ des femmes (Figure 3 ).

Figure 3. Répartition des personnes enquêtées par sexe

$\mathrm{P}$-value $=0,41357 ; \mathrm{X}^{2}=15,50731$

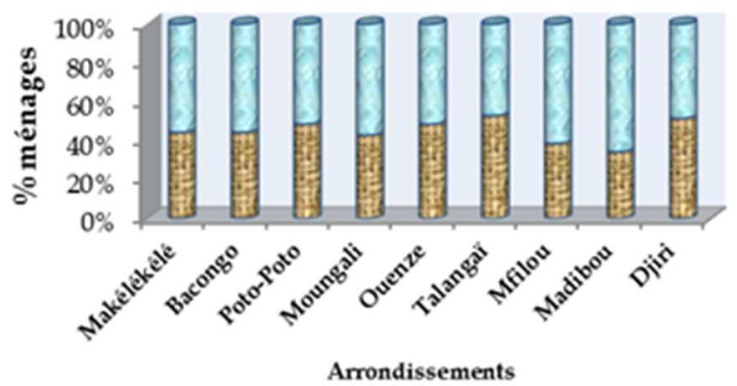

๑Masculin $\square$ Féminin 


\section{Mode d'approvisionnement en eaux}

La Figure 4 présente les types d'eaux qui sont consommés par les ménages dans les arrondissements de Brazzaville. Huit (08) types d'eaux sont consommés à savoir : l'eau de la SNDE, du forage, de puits, de pluies, des sources naturelles, de rivière, minérale, vendeur d'eau ou camion-citerne avec l'étiquette « eau potable ».

L'analyse des différentes courbes montre que, les sources d'approvisionnement en eau varient d'un arrondissement à un autre. L'eau de la SNDE et de pluies sont plus consommées dans les neuf arrondissements de Brazzaville dans les proportions respectives de 80,16 et $64,29 \%$, soit un effectif autour 50 et 70 ménages pour l'eau de pluies. Par contre, ces deux types d'eaux pris séparément sont moins utilisés dans deux arrondissements (Ouenzé et Madibou). En outre, l'eau de forage est plus consommée dans quatre arrondissements $(4,5,6$ et 7$)$ tandis que, l'eau de puits est plus utilisée dans les arrondissements 4 et 8 . L'eau des sources naturelles est plus consommée au Sud de Brazzaville dans l'arrondissement 8 (Madibou). Les eaux minérales sont moyennement utilisées par les populations dans les arrondissements Makélékélé, Bacongo, Poto-poto et M'filou.

Cependant, 10,00\% des ménages seulement consomment l'eau de pluies dans l'arrondissement 5 (Ouenzé). Cette faible consommation pourrait s'expliquer par un taux de desserte élevé (100\%) en eau du robinet. La forte consommation en eau souterraine dans l'arrondissement 8 (Madibou) serait due essentiellement à l'absence prononcée du réseau de distribution d'eau. L'enquête a révélé, 61,01 \% des ménages enregistrent des périodes de pénurie d'eau de la SNDE contre $38,99 \%$, ce phénomène est très observé $(\mathrm{P}$ $=0,00000)$ notamment dans 7 arrondissements $(1,2,3,4,6,7$ et 9). Cette pénurie serait la cause principale des diverses sources d'approvisionnement en eau des ménages dans ces dits arrondissements. Par ailleurs, les travaux réalisés par le Centre d'Etudes et de Recherche en Gestion et Entrepreneuriat, sur la pauvreté et problèmes environnementaux à Brazzaville ont montrés que, 85,50 \% des ménages ont accès à l'eau courante de la SNDE (CEREGE, 2006). 


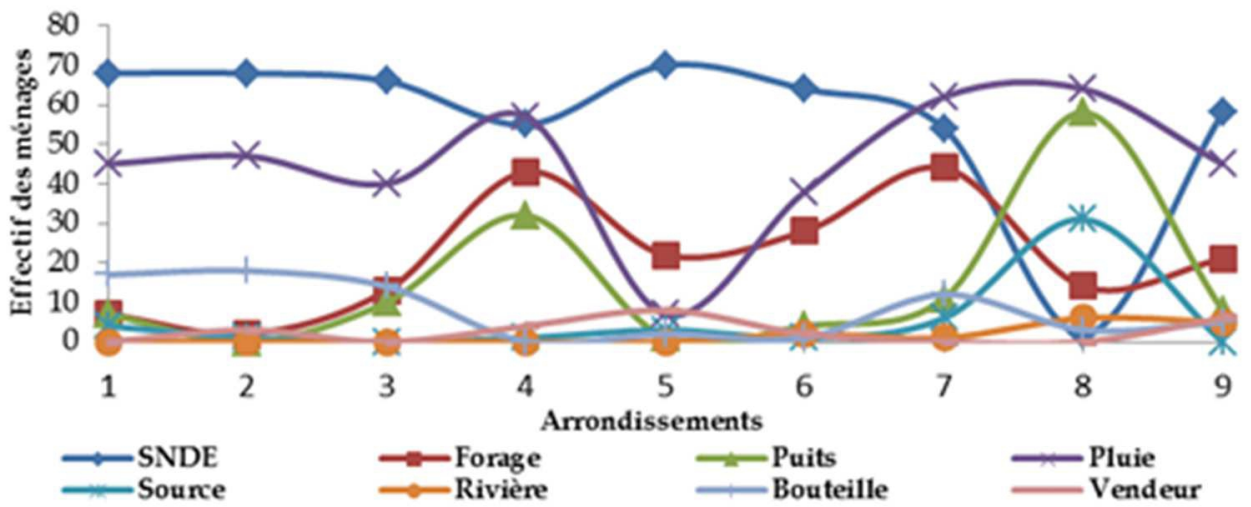

Figure 4. Types d'eaux consommés par les ménages par arrondissement

SNDE $: \mathrm{P}=0,00000, \mathrm{X}^{2}=63,67525$; Forage $: \mathrm{P}=0,00000, \mathrm{X}^{2}=80,26804$; Puits $: \mathrm{P}=$ $0,00000, \mathrm{X}^{2}=158,40458$; Eau de pluie $: \mathrm{P}=0,00000, \mathrm{X}^{2}=51,66667$; Eau de source $: \mathrm{P}=$ $0,00000, \mathrm{X}^{2}=105,65957$; Rivière $: \mathrm{P}=0,18256, \mathrm{X}^{2}=4,85714$; Eau minérale $: \mathrm{P}=0,00000$, $\mathrm{X}^{2}=38,22222$; Vendeur d'eau : $\mathrm{P}=0,20206, \mathrm{X}^{2}=9,23478$

1 : Makélékélé ; 2 : Bacongo ; 3 : Poto-poto ; 4 : Moungali ; 5 : Ouenzé ; 6 : Talangaï ; 7 : M'filou ; 8 : Madibou ; 9 : Djiri

\subsection{Distance à parcourir pour $s$ 'approvisionner en eau}

La Figure 5 présente les distances en mètre à parcourir pour s'approvisionner en eau. Il ressort, que les ménages enquêtés parcourent des distances d'au plus $100 \mathrm{~m}$ pour s'approvisionner en eau de la SNDE (9 arrondissements), de puits (Moungali et Madibou) et de forage particulièrement pour l'arrondissement 5 (Ouenzé) avec un effectif de 22 ménages (Figure 5 a). Par ailleurs, les ménages de 7 arrondissements (1, 3, 4, $6,7,8$ et 9) parcourent des distances d'au moins $101 \mathrm{~m}$ pour s'approvisionner en eau de forage et de puits. Ces résultats nous permettent de dire, que l'effectif des ménages qui s'approvisionne en eau de forage est plus élevé à Moungali (31 ménages) et M'filou (34 ménages). Concernant l'eau de puits, l'effectif le plus élevé est observé à Moungali (19 ménages). De plus, 28 ménages à Madibou parcourent une distance de plus de $101 \mathrm{~m}$ pour se ravitailler en eau de source (Figure 5b). Ainsi, l'approvisionnement en eau souterraine est une véritable corvée pour les populations de la ville de Brazzaville, particulièrement celles des arrondissements 4 (Moungali), 7 (M'filou), et 8 (Madibou). 


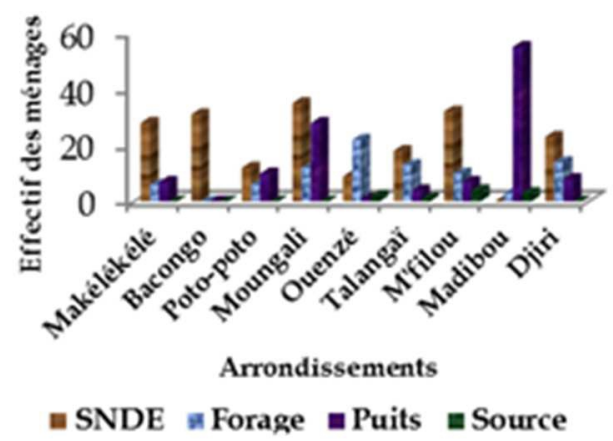

$\mathrm{a}$ : Distance d'au plus $100 \mathrm{~m}$ $\mathrm{P}=0,00000 ; \mathrm{X}^{2}=230,95027$

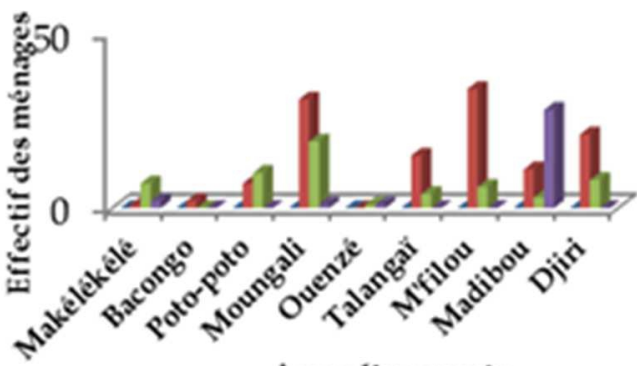

Arrondissements nSNDE $\approx$ Forage $=$ Puits $\|$ Source

b : Distance d'au moins $101 \mathrm{~m}$ $P=0,00000 ; X^{2}=143,71921$

Figure 5. Distance à parcourir pour s'approvisionner en eau

\subsection{Profondeur de l'eau de forage et de puits}

La qualité de l'eau souterraine dépend de sa profondeur et de l'hygiène autour du point d'approvisionnement (Yapo et al., 2010; Degbey et al., 2010; Coulibaly, 2005; Hassane, 2010).

Les résultats de l'enquête ont montré, que 33,33\% d'individus n'ont pas spécifiés leur réponse, $14,29 \%$ des forages présentent une profondeur autour de $50 \mathrm{~m}$ et $52,38 \%$ des forages ont une profondeur de moins de $40 \mathrm{~m}$. Par ailleurs, le puits représente $45,65 \%$ pour une profondeur de plus de $10 \mathrm{~m}$ contre $54,35 \%$ pour une profondeur inférieure à $10 \mathrm{~m}\left(\mathrm{P}=0,00000 ; \mathrm{X}^{2}=\right.$ 42,69384). Il est évident de souligner que, la ville de Brazzaville a une nappe phréatique superficielle. Les travaux de Mokolo en 1993 réalisés sur les contrôles systématiques de la qualité des eaux naturelles (souterraines) à Brazzaville ont montrés des résultats similaires (Mokolo, 1993).

\section{Perception de la qualité de l'eau : aspect des eaux consommées}

Les populations de certains arrondissements de la ville ont des inquiétudes sur la qualité des différents types d'eaux consommées. Cette inquiétude est exprimée en observant la coloration ou l'aspect de l'eau qu'elles consomment (Figure 6). L'eau de la SNDE a un aspect plus trouble dans les arrondissements Makélékélé, M'filou et Talangaï dans des proportions respectives des 95,59, 62,96 et 60,94\%. Par contre, l'aspect clair de l'eau de la SNDE est plus remarquable dans la zone Nord (Ouenzé et Djiri), au centre (Moungali et Poto-poto) et dans la zone Sud (Bacongo) de Brazzaville dans plus de 70,69 \% des ménages enquêtés (Figure 6a). Selon les données de l'enquête sur les eaux de forage et des sources naturelles, $96,90 \%$ et $97,92 \%$ respectivement des ménages ont souligné la clarté de ces eaux (Figure $6 \mathrm{~b}$ et $6 \mathrm{~d}$ ). Par ailleurs, 55,73\% des ménages enquêtés dans 
l'ensemble affirment la clarté de l'eau de puits (Figure 6c) contre 44,27\% pour l'aspect trouble. Signalons que, l'aspect trouble et coloration jaune de l'eau de la SNDE pourrait être due, d'une part, à la vétusté de la canalisation en acier, d'autre part, aux nombreuses fuites d'eau occasionnées par la perforation des nouvelles canalisations en PVC du réseau de distribution d'eau potable.

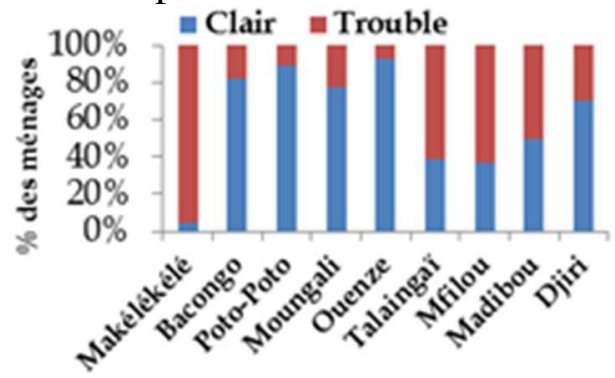

Arrondissements

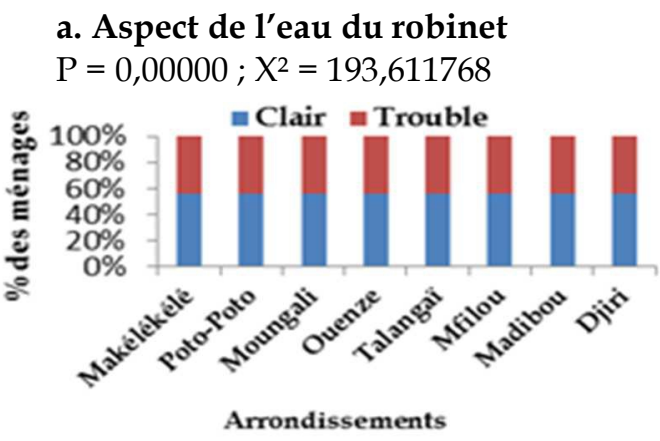

c. Aspect de l'eau de puits $\mathrm{P}=0,00000 ; \mathrm{X}^{2}=66,08899$

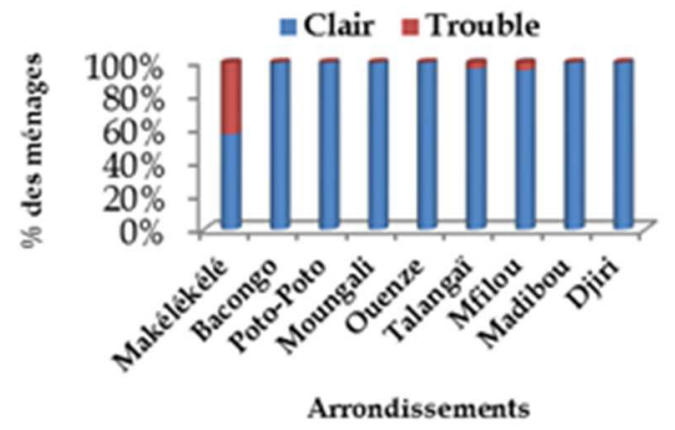

b. Aspect de l'eau de forage $P=0,00000 ; X^{2}=40,93151$

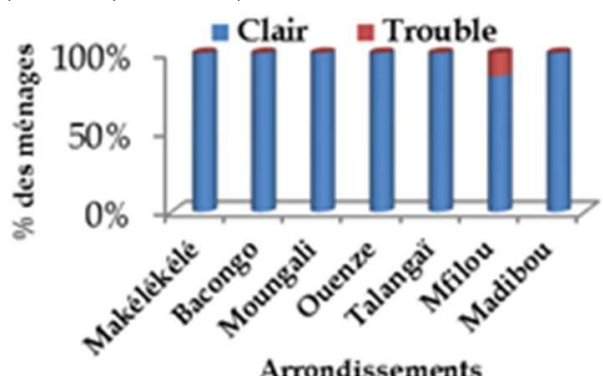

d. Aspect de l'eau de source

$P=0,00000 ; X^{2}=105,65957$

Figure 6. Aspect des eaux les plus consommées par arrondissement

\section{Evaluation de l'hygiène et de l'assainissement des ménages}

\subsection{Récipients de transport et de stockage}

Les résultats de l'enquête montrent que, les types des récipients de transport et de stockage de l'eau sont diversifiés. Les ménages utilisent presque les mêmes types des récipients pour le transport et le stockage de l'eau. Les bidons plastiques de capacité 25 litre et les seaux plastiques de contenance entre 10 à 20 litres sont plus utilisés dans les proportions respectives de $43 \%$ et $37 \%$ pour le transport, contre $45 \%$ et $39 \%$ pour le stockage. Les travaux réalisés par (Batantou, 2013) sur la problématique de l'accès à l'eau au Congo ont montré la prolifération des bidons jaunes en plastique qui envahissent la ville de Brazzaville. Cependant, ces bidons en 
plastiques présentent une ouverture trop petite pour un nettoyage efficace (Lalanne, 2012).

Pour le transport, $14 \%$ des ménages utilisent les bouteilles en plastiques préalablement vidées des eaux minérales. Les observations sur le terrain ont montré, ces bouteilles plastiques servent de transport et aussi de stockage de l'eau. Cette eau est souvent négligée et exposée à l'extérieur des maisons pour une utilisation antérieure en cas de pénurie.

Par ailleurs, les observations et l'enquête de terrain ont aussi révélés, quatre modes de transport sont souvent utilisés par les ménages: pied, brouette, voiture et pousse-pousse. $71,00 \%$ des ménages se déplacent le plus souvent à pied. La brouette, voiture et pousse-pousse sont des moyens de transport le moins utilisés dans les ménages avec une part des proportions respectives de 19,00, 3,00 et 7,00 \%. Les Figures 7a et 7b présentent les types de récipients de transport et de stockage de l'eau les plus utilisés et ceux, les moins utilisés.

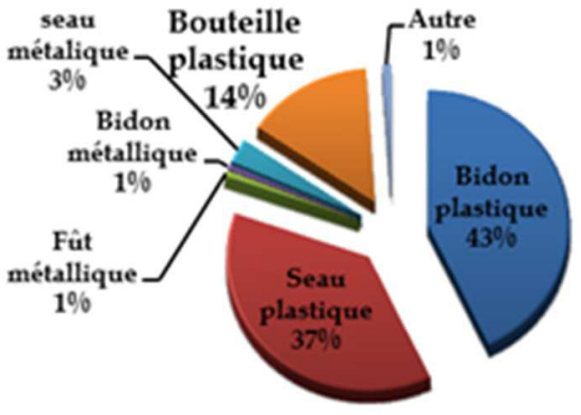

Figure 7a. Type des récipients de transport

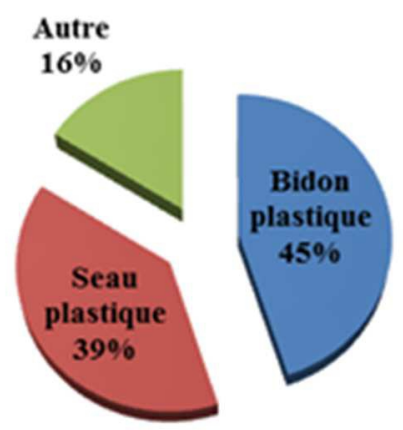

Figure 7b. Type de récipients de stockage

\subsection{Durée de conservation de l'eau}

La durée de conservation de l'eau dépend non seulement du niveau de desserte en eau des ménages par la société étatique de distribution d'eau du pays, mais aussi, des habitudes et des comportements liés aux ménages. L'analyse des résultats montre, seulement $7 \%$ des ménages conservent l'eau pendant un jour. $20 \%$ et $21 \%$ des ménages la conserve pendant deux et trois jours. D'autres par contre, conservent l'eau pendant quatre jours et plus (Figure 8). Malgré un taux élevé $(83,65 \% ; \mathrm{P}=0,00000)$ des personnes qui renouvèlent l'eau, une grande partie de la population enquêtée ignore que, la qualité de l'eau peut changer avec le temps dans l'enceinte du récipient de stockage. L'analyse de ces résultats montre, ce comportement et/ou habitude sont liés aux différentes pénuries ou coupures d'eau souvent décrié par les ménages (Lalanne, 2012). 


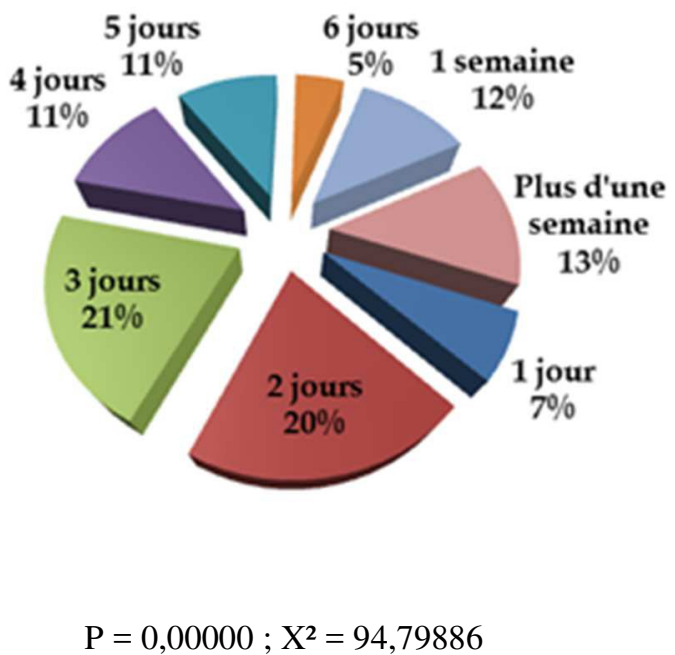

Figure 8. Durée de conservation de l'eau

\subsection{Nettoyage et objets utilisés}

Le nettoyage des récipients de transport et de conservation de l'eau à l'aide d'un détergent approprié, minimise le risque de récontamination de l'eau transportée ou stockée. Les Figures $9 \mathrm{a}$ et $9 \mathrm{~b}$ représentent la fréquence de nettoyage et les produits utilisés pour le nettoyage.

La population enquêtée dans sa presque totalité $(98 \% ; \mathrm{P}=0,00000)$ affirme nettoyer les récipients utilisés tant pour le transport que pour le stockage de l'eau. Elle est consciente qu'un nettoyage régulier avant le remplissage $(88 \%)$ du récipient est important pour maintenir la qualité de l'eau d'origine (Figure 15a). Cependant, $35 \%, 31 \%, 11 \%, 6 \%, 13 \%$ et 4 $\%$ des ménages utilisent respectivement l'eau simple, savon, détergent (pax), détergent (javel) et autre pour nettoyer les récipients (Figure 15b). Nous avons constaté que le nettoyage n'est pas efficace, car $13 \%$ seulement des ménages pratiquent le nettoyage avec la javel qui est un agent désinfectant.

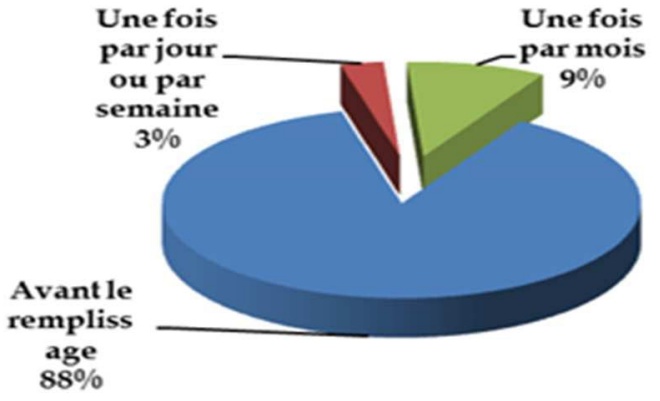

Figure 9a. Fréquence de nettoyage $\mathrm{P}=0,00000 ; \mathrm{X}^{2}=820,16585$

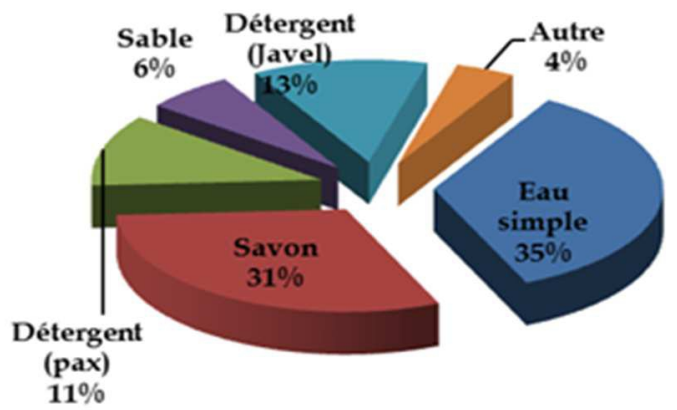

Figure 9b. Part des proportions des objets de nettoyage 


\subsection{Utilisation et technique de traitement de l'eau à domicile}

La connaissance des techniques de traitement de l'eau à domicile est très importante pour les populations des zones urbaines et rurales. Les Figures $10 \mathrm{a}$ et $10 \mathrm{~b}$ présentent respectivement la connaissance ou non et les techniques de traitement de l'eau à domicile. 318 (50,5\%) ménages affirment pratiquer le traitement de l'eau à domicile contre $312(49,5 \%)$ qui n'en pratiquent pas (Figure 10a). Certains enquêtés, affirment avoir reçu une formation de la part de la croix rouge congolaise et d'autres Organismes Non Gouvernementaux (ONG) de la ville. Cependant, les observations de terrain ont montré, que la plus grande partie de la population enquêtée qui prétend traiter l'eau à domicile ne maitrise pas la quantité de produit qu'il faut mettre dans un certain volume d'eau pour l'eau de Javel. Cette population ignore qu'il existe un degré chlorométrique qu'il faut prendre en compte dans l'eau de Javel.

Par ailleurs, les techniques de traitement utilisées sont essentiellement la filtration (26\%), la désinfection à l'eau de Javel (21\%), le chauffage (28 $\%)$, la décantation (10\%), et la désinfection à la Javel en grain (15\%) (Figure 10b). Nous constatons que, la technique de chauffage et de filtration de l'eau sont plus utilisées par les ménages. Car les ménages enquêtés, affirment la facilité d'utiliser le filtre moderne vendu au marché et trouvent contraignant la méthode du chauffage à cause de la durée du chauffage et de refroidissement.

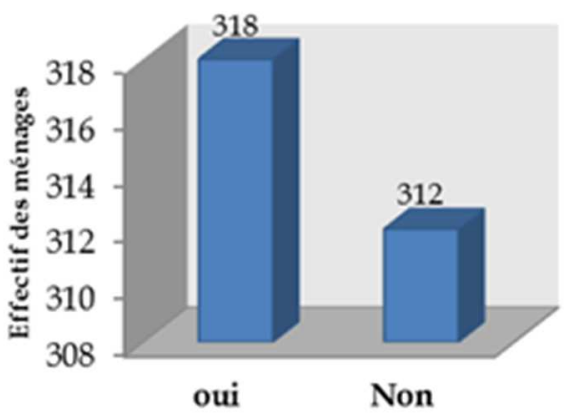

Figure 10a. Utilisation ou non des techniques de traitement de l'eau $\mathrm{P}=0,81107 ; \mathrm{X}^{2}=0,05714$

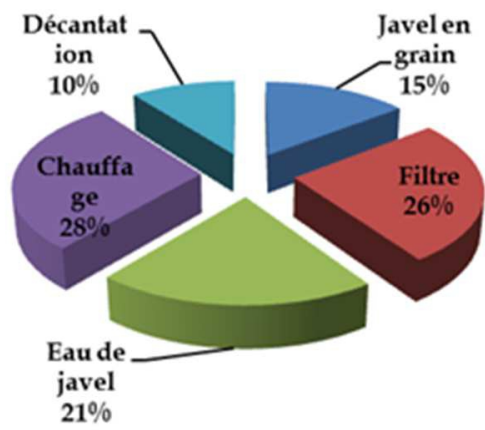

Figure 10a. Part des proportions des techniques de traitement de l'eau à domicile

\section{Conclusion}

Cette étude montre que, les personnes dont la tranche d'âge se situe entre 21 à 30 ans et 31 à 40 ans ont été dominantes pendant le sondage avec des taux respectifs de $36,35 \%$ et $23,65 \%$. Les femmes représentent $54,92 \%$ contre 45,08 \% des hommes. Malgré un taux de desserte élevé (78 \%) de la 
Société Nationale de Distribution d'Eau (SNDE) dans la ville de Brazzaville, cette société ne satisfait pas pleinement au besoin de la population. Elle doute de la qualité de l'eau produite et distribuée de par son aspect et la vétusté de ces installations. Les conditions d'hygiène et d'assainissement sont précaires dans les ménages et dans les endroits les plus fréquentés de la ville. Les mauvaises pratiques de transport et de stockage des eaux par les populations les exposent à une contamination et récontamination. Cette étude a été menée afin de guider les décideurs dans la gestion d'approvisionnement en eaux, d'assainissement et d'hygiène dans la ville de Brazzaville.

\section{References:}

1. Batantou O. (2013). Problématique de l'accès à l'eau potable au Congo. Alternatives citoyennes. Bimestriel d'information et d'échanges du Programme Concerté Pluri-Acteurs - PCPA-Congo, 16 p.

2. Boubakar Hassane A. (2010). Aquifères superficiels et profonds et pollution urbaine en Afrique : Cas de la communauté urbaine de Niamey (NIGER), Thèse de doctorat, Université Abdou Moumouni de Niamey (Niger), 198 p.

3. Centre d'Etudes et de Recherche en Gestion et Entrepreneuriat (CEREGE) (2006). Brazzaville. Pauvreté et problèmes environnementaux. Programme international de recherche sur les interactions entre la population, le développement et l'environnement (PRIPODE). Rapport final, 149 p.

4. Centre d'Etudes et de Recherche sur les Analyses et Politiques Economiques (CERAPE). $6^{\text {ème }}$ Conférence Africaine sur la Population, Ouagadougou, $19 \mathrm{p}$.

5. Coulibaly K. (2005). Étude de la qualité physico-chimique et bactériologique de l'eau de puits de certains quartiers du district de Bamako. Thèse de Doctorat en Pharmacie, Faculté de Médecine de Pharmacie et d'Odonto-Stomatologie, Université de Bamako, 69 p.

6. Degbey C., Makoutode M., \& de Brouwer C. (2010). La qualité de l'eau de boisson en milieu professionnel à Godomey en 2009 au Bénin Afrique de l'Ouest, J Int Santé Trav., 1 : 15-22.

7. El Kharmouz M., Sbaa M., Chafi A., \& Saadi S. (2013). L'étude de l'impact des lixiviats de l'ancienne décharge publique de la ville d'Oujda (Maroc oriental) sur la qualité physicochimique des eaux souterraines et superficielles. Larhyss Journal, 16 : 105-119.

8. Koua O.J. \& Nzaou S. (2011). Perspective de la population congolaise de 2007 à 2020, CNSEE, 11 p.

9. Kouame K.V. \& Houenou P. (2010). Évaluation de la qualité des eaux de puits à usage domestique dans les quartiers défavorisés de 
quatre communes d'Abidjan (Côte d'Ivoire) : Koumassi, Marcory, Port-Bouet et Treichville Int., J. Biol. Chem. Sci., 4(2) : 289-307.

10. Lalanne F. (2012). Etude de la qualité de l'eau le long de la chaîne d'approvisionnement au niveau des consommateurs dans 10 villages de la Province du Ganzourgou, (Région du Plateau Central, Burkina Faso). 2iE, UNICEF, Burikna Faso, 71 p.

11. Makouézi M.C. \& Moboula E.J. (2011). Tendances et conséquences de l'urbanisation du Congo et de la République Démocratique du Congo (RDC).

12. Moukolo N. (1993). Les contrôles systématiques de la qualité des Eaux Naturelles $\mathrm{Au}$ Congo : Quelques résultats du laboratoire d'hydrologie de l'ORSTOM/DGRST de Brazzaville. Bulletin de liaison du CIEH n ${ }^{\circ} 92,12 \mathrm{p}$.

13. Ndinga-Okina S. (2008). Contribution à l'amélioration des voiries urbaines de Brazzaville, mémoire de Master, Université Marien Ngouabi, Faculté des Lettres et des Sciences Humaines, 53 p.

14. OMS (2010). Études méthodologiques. Guide pratique pour la conception d'enquêtes sur les ménages. Nations Unies, New York, 264 p.

15. OMS \& UNICEF (2007). Atteindre l'Objectif du Millénaire pour le Développement relatif à l'eau potable et à l'assainissement: le défi urbain et rural de la décennie. UN Plaza, New York, NY 10017, Etats-Unis d'Amérique, $48 \mathrm{p}$.

16. Statistique Canada (2003). Méthodes et pratiques d'enquête, No 12587-X, ISBN 978-1-100-95206-2, hors-série, 434 p.

17. Yapo O.B., Mambo V., Seka A., Ohou M.J.A., Konan F., Gouzile V., Tidou A.S.,\& Lalanne F. (2012). Etude de la qualité de l'eau le long de la chaîne d'approvisionnement au niveau des consommateurs dans 10 villages de la Province du Ganzourgou, (Région du Plateau Central, Burkina Faso). 2iE, UNICEF, Burikna Faso, 71 p. 


\section{ANNEXE}

\section{Tableaux statistiques}

1-Caractéristiques sociodémographiques

Tableau I. Groupe d'âge par arrondissement (correspond à la Figure 2)

\begin{tabular}{|c|c|c|c|c|c|c|c|c|c|c|c|c|c|c|c|c|c|c|c|c|c|}
\hline \multirow{3}{*}{$\begin{array}{c}\text { Group } \\
\text { e } \\
\text { d'âges }\end{array}$} & \multicolumn{19}{|c|}{ Arrondissements } & \multirow{2}{*}{\multicolumn{2}{|c|}{ Total }} \\
\hline & \multicolumn{2}{|c|}{$\begin{array}{l}\text { Makélé } \\
\text { kélé }\end{array}$} & \multicolumn{2}{|c|}{ Bacongo } & \multicolumn{2}{|c|}{$\begin{array}{l}\text { Poto- } \\
\text { Poto }\end{array}$} & \multicolumn{2}{|c|}{$\begin{array}{c}\text { Moung } \\
\text { ali }\end{array}$} & \multicolumn{2}{|c|}{ Ouenzé } & \multicolumn{3}{|c|}{$\begin{array}{c}\text { Talanga } \\
\text { ï }\end{array}$} & \multicolumn{2}{|c|}{ M'filou } & \multicolumn{2}{|c|}{$\begin{array}{c}\text { Madibo } \\
\mathrm{u}\end{array}$} & \multicolumn{2}{|c|}{ Djiri } & & \\
\hline & Eff & $\%$ & Eff & $\%$ & $E$ & $\%$ & $\begin{array}{l}\text { Ef } \\
f .\end{array}$ & $\%$ & & $\%$ & & & & Eff & $\%$ & Eff & $\%$ & Eff & $\%$ & Eff. & $\%$ \\
\hline $\begin{array}{l}\mathrm{Au} \\
\text { lus } 20 \\
\text { ans }\end{array}$ & 5 & $\begin{array}{c}7,1 \\
4\end{array}$ & 2 & 36 & 9 & $\begin{array}{c}12,8 \\
6\end{array}$ & $\begin{array}{l}1 \\
2\end{array}$ & $\begin{array}{l}17, \\
14\end{array}$ & 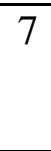 & $\begin{array}{c}10,0 \\
0\end{array}$ & & & & & $\begin{array}{l}14, \\
29\end{array}$ & & $\begin{array}{c}7,1 \\
4\end{array}$ & 16 & 6 & 2 & $\begin{array}{c}14,6 \\
0\end{array}$ \\
\hline $\begin{array}{l}\mathrm{De} 2 \\
\text { à } 3\end{array}$ & 30 & $\begin{array}{c}42, \\
9\end{array}$ & 27 & $\begin{array}{c}38,5 \\
7\end{array}$ & 9 & $\begin{array}{c}41,4 \\
3\end{array}$ & 6 & $\begin{array}{l}37, \\
14\end{array}$ & & $\begin{array}{c}40,0 \\
0\end{array}$ & & & & 26 & $\begin{array}{l}37, \\
14\end{array}$ & 17 & $\begin{array}{l}24, \\
29\end{array}$ & 23 & 2, & 29 & $\begin{array}{c}36,3 \\
5\end{array}$ \\
\hline $\begin{array}{c}\text { De } 31 \\
\text { à } 40 \\
\text { ans }\end{array}$ & 22 & $\begin{array}{c}31, \\
4\end{array}$ & 25 & $\begin{array}{c}35,7 \\
1\end{array}$ & $\begin{array}{l}1 \\
3\end{array}$ & $\begin{array}{c}18,5 \\
7\end{array}$ & 7 & \begin{tabular}{|l|}
24, \\
29
\end{tabular} & & $\begin{array}{c}17,1 \\
4\end{array}$ & & & & 18 & $\begin{array}{c}25 \\
71\end{array}$ & 16 & $\begin{array}{l}22, \\
86\end{array}$ & 14 & $\begin{array}{l}20, \\
00\end{array}$ & 149 & $\begin{array}{c}23,6 \\
5\end{array}$ \\
\hline $\begin{array}{c}\text { De } 41 \\
\text { à } 50 \\
\text { ans }\end{array}$ & 7 & 10 & 12 & $\begin{array}{c}17,1 \\
4\end{array}$ & 7 & $\begin{array}{c}10,0 \\
0\end{array}$ & 9 & $\begin{array}{l}12 \\
86\end{array}$ & & $\begin{array}{c}20,0 \\
0\end{array}$ & 4 & & & 6 & $\begin{array}{c}8,5 \\
7\end{array}$ & 17 & $\begin{array}{l}24, \\
29\end{array}$ & 9 & $\begin{array}{l}12, \\
86\end{array}$ & 8. & $\begin{array}{c}13,4 \\
9\end{array}$ \\
\hline $\begin{array}{c}\mathrm{Au} \\
\text { moins }\end{array}$ & 6 & $\begin{array}{c}8,5 \\
7\end{array}$ & 4 & 5,71 & $\begin{array}{l}1 \\
2\end{array}$ & $\begin{array}{c}17,1 \\
4\end{array}$ & 6 & $\begin{array}{c}8,5 \\
7\end{array}$ & 9 & $\begin{array}{c}12,8 \\
6\end{array}$ & $J$ & & & 10 & $\begin{array}{l}14, \\
29\end{array}$ & 15 & $\begin{array}{l}21, \\
43\end{array}$ & 8 & $\begin{array}{l}11, \\
43\end{array}$ & 75 & $\begin{array}{c}11,9 \\
0\end{array}$ \\
\hline Total & 70 & 10 & 10 & 100 & & 100 & 1 & 10 & & 100 & & & 00 & 70 & 100 & 70 & 10 & 70 & 10 & 630 & 100 \\
\hline
\end{tabular}


European Scientific Journal July 2017 edition Vol.13, No.21 ISSN: 1857 - 7881 (Print) e - ISSN 1857- 7431

\begin{tabular}{|l|l|l|l|l|l|l|l|l|l|l|l|l|l|l|l|l|l|l|l|l|}
\hline & & 0 & & & 0 & & 0 & 0 & 0 & & 0 & & & & & 0 & & 0 & & \\
\hline
\end{tabular}

Tableau II. Sexe des répondants par arrondissement (correspond à la Figure 3)

\begin{tabular}{|c|c|c|c|c|}
\hline \multirow{2}{*}{ Arrondissements } & \multicolumn{3}{|c|}{ Sexe des enquêtés } & Total \\
\cline { 2 - 5 } & & Masculin & Féminin & \\
\hline \multirow{2}{*}{ Makélékélé } & Eff. & 31 & 39 & 70 \\
\cline { 2 - 5 } & $\%$ & 44,29 & 55,71 & 100 \\
\hline \multirow{2}{*}{ Bacongo } & Eff. & 31 & 39 & 70 \\
\cline { 2 - 5 } & $\%$ & 44,29 & 55,71 & 100 \\
\hline \multirow{2}{*}{ Poto-Poto } & Eff. & 34 & 36 & 70 \\
\cline { 2 - 5 } & $\%$ & 48,57 & 51,43 & 100 \\
\hline \multirow{2}{*}{ Moungali } & Eff. & 30 & 40 & 70 \\
\cline { 2 - 5 } & $\%$ & 42,86 & 57,14 & 100 \\
\hline \multirow{2}{*}{ Ouenzé } & Eff. & 34 & 36 & 70 \\
\cline { 2 - 5 } & $\%$ & 48,57 & 51,43 & 100 \\
\hline \multirow{2}{*}{ Talangaï } & Eff. & 37 & 33 & 70 \\
\cline { 2 - 5 } & $\%$ & 52,86 & 47,14 & 100 \\
\hline \multirow{2}{*}{ M'filou } & Eff. & 27 & 43 & 70 \\
\cline { 2 - 5 } & $\%$ & 38,57 & 61,43 & 100 \\
\hline \multirow{2}{*}{ Madibou } & Eff. & 24 & 46 & 70 \\
\cline { 2 - 5 } & $\%$ & 34,29 & 65,71 & 100 \\
\hline \multirow{2}{*}{ Djiri } & Eff. & 36 & 34 & 70 \\
\cline { 2 - 5 } & $\%$ & 51,43 & 48,57 & 100 \\
\hline \multirow{2}{*}{ Total } & Eff. & 284 & 346 & 630 \\
\cline { 2 - 5 } & $\%$ & 45,08 & 54,92 & 100 \\
\hline
\end{tabular}




\section{Eff. $=$ Effectif $; \%=$ Pourcentage}

1. Mode d'approvisionnement en eaux

Tableau III. Types d'eaux consommés par les ménages par arrondissement (correspond à la Figure 4)

\begin{tabular}{|c|c|c|c|c|c|c|c|c|c|c|c|c|c|c|c|c|c|c|c|c|}
\hline \multirow{2}{*}{$\begin{array}{c}\text { Arr } \\
\text { Nature } \\
\text { d'eaux }\end{array}$} & \multicolumn{2}{|c|}{$\begin{array}{l}\text { Makéléké } \\
\text { lé }\end{array}$} & \multicolumn{2}{|c|}{ Bacongo } & \multicolumn{2}{|c|}{$\begin{array}{l}\text { Poto- } \\
\text { Poto }\end{array}$} & \multicolumn{2}{|c|}{$\begin{array}{c}\text { Moungal } \\
\mathrm{i}\end{array}$} & \multicolumn{2}{|c|}{ Ouenzé } & \multicolumn{2}{|c|}{ Talangaï } & \multicolumn{2}{|c|}{ M'filou } & \multicolumn{2}{|c|}{ Madibou } & \multicolumn{2}{|c|}{ Djiri } & \multicolumn{2}{|c|}{ Total } \\
\hline & Eff & Prop & Eff & Prop & Eff & Prop & Eff & Prop & Eff & Prop & Eff & Prop & Eff & Prop & Eff & Prop & Eff & Prop & Eff & Prop \\
\hline SNDE & 68 & $\begin{array}{c}97,1 \\
4\end{array}$ & 68 & $\begin{array}{c}97,1 \\
4\end{array}$ & 66 & $\begin{array}{c}94,2 \\
9\end{array}$ & 55 & $\begin{array}{c}78,5 \\
7\end{array}$ & 70 & $\begin{array}{c}100,0 \\
0\end{array}$ & 64 & $\begin{array}{c}91,4 \\
3\end{array}$ & 54 & $\begin{array}{c}77,1 \\
4\end{array}$ & 2 & 2,86 & 58 & $\begin{array}{c}82,8 \\
6\end{array}$ & $\begin{array}{c}50 \\
5\end{array}$ & 80,16 \\
\hline Forage & 7 & $\begin{array}{c}10,0 \\
0 \\
\end{array}$ & 2 & 2,86 & 13 & $\begin{array}{c}18,5 \\
7 \\
\end{array}$ & 43 & $\begin{array}{c}61,4 \\
3 \\
\end{array}$ & 22 & 31,43 & 28 & $\begin{array}{c}40,0 \\
0 \\
\end{array}$ & 44 & $\begin{array}{c}62,8 \\
6 \\
\end{array}$ & 14 & $\begin{array}{c}20,0 \\
0\end{array}$ & 21 & $\begin{array}{c}30,0 \\
0 \\
\end{array}$ & $\begin{array}{c}19 \\
4 \\
\end{array}$ & 30,79 \\
\hline Puits & 7 & $\begin{array}{c}10,0 \\
0 \\
\end{array}$ & 0 & 0,00 & 10 & $\begin{array}{c}14,2 \\
9 \\
\end{array}$ & 32 & $\begin{array}{c}45,7 \\
1 \\
\end{array}$ & 1 & 1,43 & 4 & 5,71 & 11 & $\begin{array}{c}15,7 \\
1 \\
\end{array}$ & 58 & $\begin{array}{c}82,8 \\
6 \\
\end{array}$ & 8 & $\begin{array}{c}11,4 \\
3 \\
\end{array}$ & $\begin{array}{c}13 \\
1 \\
\end{array}$ & 20,79 \\
\hline Pluie & 45 & $\begin{array}{c}64,2 \\
9 \\
\end{array}$ & 47 & $\begin{array}{c}67,1 \\
4\end{array}$ & 40 & $\begin{array}{c}57,1 \\
4 \\
\end{array}$ & 57 & $\begin{array}{c}81,4 \\
3\end{array}$ & 7 & 10,00 & 38 & $\begin{array}{c}54,2 \\
9 \\
\end{array}$ & 62 & $\begin{array}{c}88,5 \\
7\end{array}$ & 64 & $\begin{array}{c}91,4 \\
3\end{array}$ & 45 & $\begin{array}{c}64,2 \\
9\end{array}$ & $\begin{array}{c}40 \\
5\end{array}$ & 64,29 \\
\hline Source & 4 & 5,71 & 1 & 1,43 & 0 & 0,00 & 1 & 1,43 & 3 & 4,29 & 1 & 1,43 & 6 & 8,57 & 31 & $\begin{array}{c}44,2 \\
9\end{array}$ & 0 & 0,00 & 47 & 7,46 \\
\hline Rivière & 0 & 0,00 & 0 & 0,00 & 0 & 0,00 & 0 & 0,00 & 0 & 0,00 & 2 & 2,86 & 1 & 1,43 & 6 & 8,57 & 5 & 7,14 & 14 & 2,22 \\
\hline $\begin{array}{c}\text { Bouteil } \\
\text { le }\end{array}$ & 17 & $\begin{array}{c}24,2 \\
9 \\
\end{array}$ & 18 & $\begin{array}{c}25,7 \\
1 \\
\end{array}$ & 14 & $\begin{array}{c}20,0 \\
0 \\
\end{array}$ & 0 & 0,00 & 2 & 2,86 & 1 & 1,43 & 12 & $\begin{array}{c}17,1 \\
4 \\
\end{array}$ & 3 & 4,29 & 5 & 7,14 & 71 & 11,27 \\
\hline $\begin{array}{c}\text { Vendeu } \\
\mathrm{r}\end{array}$ & 0 & 0,00 & 3 & 4,29 & 0 & 0,00 & 4 & 5,71 & 8 & 11,43 & 2 & 2,86 & 0 & 0,00 & 0 & 0,00 & 6 & 9 & 23 & 3,65 \\
\hline
\end{tabular}

Eff. $=$ Effectif $;$ Prop $=$ Proportion $;$ Arr : Arrondissements 
1.1. Distance à parcourir pour s'approvisionner en eau

Tableau IV. Distance d'au plus $100 \mathrm{~m}$ à parcourir pour s'approvisionner en eau (correspond à la Figure 5a)

\begin{tabular}{|c|c|c|c|c|c|c|c|c|c|c|}
\hline \multirow{2}{*}{ Nature d'eaux } & \multicolumn{2}{|c|}{ SNDE } & \multicolumn{2}{|c|}{ Forage } & \multicolumn{2}{c|}{ Puits } & \multicolumn{2}{c|}{ Source } & \multicolumn{2}{c|}{ Total } \\
\cline { 2 - 15 } & Eff. & $\%$ & Eff. & $\%$ & Eff. & $\%$ & Eff. & $\%$ & Eff. & $\%$ \\
\hline Makélékélé & 28 & 14,89 & 6 & 6,98 & 7 & 5,83 & 0 & 0,00 & 41 & 10,15 \\
\hline Bacongo & 31 & 16,49 & 0 & 0,00 & 0 & 0,00 & 0 & 0,00 & 31 & 7,67 \\
\hline Poto-poto & 12 & 6,38 & 6 & 6,98 & 10 & 8,33 & 0 & 0,00 & 28 & 6,93 \\
\hline Moungali & 35 & 18,62 & 12 & 13,95 & 28 & 23,33 & 0 & 0,00 & 75 & 18,56 \\
\hline Ouenzé & 9 & 4,79 & 22 & 25,58 & 1 & 0,83 & 2 & 20,00 & 34 & 8,42 \\
\hline Talangaï & 18 & 9,57 & 13 & 15,12 & 4 & 3,33 & 1 & 10,00 & 36 & 8,91 \\
\hline M'filou & 32 & 17,02 & 10 & 11,63 & 7 & 5,83 & 4 & 40,00 & 53 & 13,12 \\
\hline Madibou & 0 & 0,00 & 3 & 3,49 & 55 & 45,83 & 3 & 30,00 & 61 & 15,10 \\
\hline Djiri & 23 & 12,23 & 14 & 16,28 & 8 & 6,67 & 0 & 0,00 & 45 & 11,14 \\
\hline Total & 188 & 100 & 86 & 100 & 120 & 100 & 10 & 100 & 404 & 100 \\
\hline
\end{tabular}

Eff. $=$ Effectif $; \%=$ Pourcentage 
Tableau IV. Distance de $101 \mathrm{~m}$ et plus à parcourir pour s'approvisionner en eau (correspond à la Figure 5b)

\begin{tabular}{|c|c|c|c|c|c|c|c|c|c|c|}
\hline \multirow{2}{*}{ Nature d'eaux } & \multicolumn{2}{|c|}{ SNDE } & \multicolumn{2}{|c|}{ Forage } & \multicolumn{2}{c|}{ Puits } & \multicolumn{2}{c|}{ Source } & \multicolumn{2}{c|}{ Total } \\
\cline { 2 - 15 } & Eff. & $\%$ & Eff. & $\%$ & Eff. & $\%$ & Eff. & $\%$ & Eff. & $\%$ \\
\hline Makélékélé & 0 & 0 & 1 & 0,82 & 7 & 12,07 & 2 & 6,25 & 10 & 4,72 \\
\hline Bacongo & 0 & 0 & 2 & 1,64 & 0 & 0,00 & 0 & 0,00 & 2 & 0,94 \\
\hline Poto-poto & 0 & 0 & 7 & 5,74 & 10 & 17,24 & 0 & 0,00 & 17 & 8,02 \\
\hline Moungali & 0 & 0 & 31 & 25,41 & 19 & 32,76 & 1 & 3,13 & 51 & 24,06 \\
\hline Ouenzé & 0 & 0 & 0 & 0,00 & 1 & 1,72 & 1 & 3,13 & 2 & 0,94 \\
\hline Talangaï & 0 & 0 & 15 & 12,30 & 4 & 6,90 & 0 & 0,00 & 19 & 8,96 \\
\hline M'filou & 0 & 0 & 34 & 27,87 & 6 & 10,34 & 0 & 0,00 & 40 & 18,87 \\
\hline Madibou & 0 & 0 & 11 & 9,02 & 3 & 5,17 & 28 & 87,50 & 42 & 19,81 \\
\hline Djiri & 0 & 0 & 21 & 17,21 & 8 & 13,79 & 0 & 0,00 & 29 & 13,68 \\
\hline Total & 0 & 0 & 122 & 100 & 58 & 100 & 32 & 100 & 212 & 100 \\
\hline
\end{tabular}

Eff. $=$ Effectif $; \%=$ Pourcentage

1.2. Profondeur de l'eau de forage et de puits

Tableau V. Profondeur de l'eau de forage

\begin{tabular}{|c|c|c|c|c|c|c|c|c|c|c|c|c|}
\hline \multirow{2}{*}{$\begin{array}{l}\text { Arrondissements } \\
\text { Profondeur }\end{array}$} & \multicolumn{2}{|c|}{ Moungali } & \multicolumn{2}{|c|}{ Ouenzé } & \multicolumn{2}{|c|}{ Talangaï } & \multicolumn{2}{|c|}{ M'filou } & \multicolumn{2}{|c|}{ Djiri } & \multicolumn{2}{|c|}{ Total } \\
\hline & Eff. & $\%$ & Eff. & $\%$ & Eff. & $\%$ & Eff. & $\%$ & Eff. & $\%$ & Eff. & $\%$ \\
\hline NSP & 0 & 0,00 & 2 & 9,52 & 4 & 19,05 & 1 & 4,76 & 1 & 4,76 & 7 & 33,33 \\
\hline Moins de $40 \mathrm{~m}$ & 5 & 23,81 & 1 & 4,76 & 1 & 4,76 & 2 & 9,52 & 2 & 9,52 & 11 & 52,38 \\
\hline
\end{tabular}




\begin{tabular}{|l|l|l|l|l|l|l|l|l|l|l|l|l|}
\hline Autour de $50 \mathrm{~m}$ & 0 & 0,00 & 0 & 0,00 & 1 & 4,76 & 2 & 9,52 & 0 & 0,00 & 3 & 14,29 \\
\hline
\end{tabular}

Eff. $=$ Effectif $; \%=$ Pourcentage $;$ NSP : Réponse non spécifiée

Tableau VI. Profondeur de l'eau de puits

\begin{tabular}{|c|c|c|c|c|c|c|c|c|c|c|c|c|c|c|c|c|}
\hline \multirow{2}{*}{$\begin{array}{c}\begin{array}{c}\text { Arrondissements } \\
\text { Profondeur }\end{array} \\
\end{array}$} & \multicolumn{2}{|c|}{ Makélékélé } & \multicolumn{2}{|c|}{ Poto-Poto } & \multicolumn{2}{|c|}{ Moungali } & \multicolumn{2}{|c|}{ Talaingaï } & \multicolumn{2}{|c|}{ Mfilou } & \multicolumn{2}{|c|}{ Madibou } & \multicolumn{2}{|c|}{ Djiri } & \multicolumn{2}{|c|}{ Total } \\
\hline & Eff. & $\%$ & Eff. & $\%$ & Eff. & $\%$ & Eff. & $\%$ & Eff. & $\%$ & Eff. & $\%$ & Eff. & $\%$ & Eff. & $\%$ \\
\hline Moins de $10 \mathrm{~m}$ & 6 & 100,00 & 8 & 100,00 & 14 & 93,33 & 3 & 75,00 & 2 & 100,00 & 13 & 25,00 & 4 & 80,00 & 50 & 54,35 \\
\hline $10 \mathrm{~m}$ et plus & 0 & 0,00 & 0 & 0,00 & 1 & 6,67 & 1 & 25,00 & 0 & 0,00 & 39 & 75,00 & 1 & 20,00 & 42 & 45,65 \\
\hline
\end{tabular}

2. Perception de la qualité de l'eau : aspect des eaux consommées (correspond à la Figure 6)

Tableau VII. Aspect des eaux les plus consommées par arrondissement

\begin{tabular}{|c|c|c|c|c|c|c|c|c|c|c|c|c|c|c|c|c|c|c|c|c|}
\hline \multirow{3}{*}{$\begin{array}{l}\text { Arr } \\
\text { Aspec } \\
\text { t des } \\
\text { eaux }\end{array}$} & \multicolumn{18}{|c|}{ Eau de la Société Nationale de Distribution d'eau (SNDE) } & \multicolumn{2}{|c|}{ Total } \\
\hline & \multicolumn{2}{|c|}{$\begin{array}{c}\text { Makéléké } \\
\text { lé }\end{array}$} & \multicolumn{2}{|c|}{ Bacongo } & \multicolumn{2}{|c|}{$\begin{array}{l}\text { Poto- } \\
\text { Poto } \\
\end{array}$} & \multicolumn{2}{|c|}{ Moungali } & \multicolumn{2}{|c|}{ Ouenze } & \multicolumn{2}{|c|}{ Talangaï } & \multicolumn{2}{|c|}{ Mfilou } & \multicolumn{2}{|c|}{ Madibou } & \multicolumn{2}{|c|}{ Djiri } & \multirow[b]{2}{*}{ Eff. } & \multirow[b]{2}{*}{$\%$} \\
\hline & Eff & $\%$ & Eff & $\%$ & Eff & $\%$ & Eff. & $\%$ & Eff. & $\%$ & Eff. & $\%$ & Eff. & $\%$ & Eff. & $\%$ & Eff. & $\%$ & & \\
\hline Clair & 3 & 4,41 & 56 & $\begin{array}{l}82, \\
35 \\
\end{array}$ & 59 & $\begin{array}{c}89,3 \\
9\end{array}$ & 43 & $\begin{array}{c}78,1 \\
8\end{array}$ & 65 & $\begin{array}{c}92,8 \\
6\end{array}$ & 25 & $\begin{array}{c}39,0 \\
6\end{array}$ & 20 & 37,04 & 1 & 50 & 41 & 70,69 & 313 & 61,98 \\
\hline $\begin{array}{c}\text { Troub } \\
\text { le }\end{array}$ & 65 & $\begin{array}{c}95,5 \\
9\end{array}$ & 12 & $\begin{array}{l}17 \\
65\end{array}$ & 7 & $\begin{array}{c}10,6 \\
1\end{array}$ & 12 & $\begin{array}{c}21,8 \\
2\end{array}$ & 5 & 7,14 & 39 & $\begin{array}{c}60,9 \\
4\end{array}$ & 34 & 62,96 & 1 & 50 & 17 & 29,31 & 192 & 38,02 \\
\hline Total & 68 & 100 & 68 & 100 & 66 & 100 & 55 & 100 & 70 & 100 & 64 & 100 & 54 & 100 & 2 & 100 & 58 & 100 & 505 & 100 \\
\hline \multicolumn{21}{|c|}{ Eau de forage } \\
\hline Clair & 4 & $\begin{array}{c}57,1 \\
4 \\
\end{array}$ & 2 & 100 & 13 & 100 & 43 & 100 & 22 & 100 & 27 & \begin{tabular}{|c|}
96,4 \\
3 \\
\end{tabular} & 42 & 95,45 & 14 & 100 & 21 & 100 & 188 & 96,91 \\
\hline
\end{tabular}




\begin{tabular}{|c|c|c|c|c|c|c|c|c|c|c|c|c|c|c|c|c|c|c|c|c|}
\hline $\begin{array}{c}\text { Troub } \\
\text { le }\end{array}$ & 3 & $\begin{array}{c}42,8 \\
6\end{array}$ & 0 & 0 & 0 & 0 & 0 & 0 & 0 & 0 & 1 & 3,57 & 2 & 4,55 & 0 & 0 & 0 & 0 & 6 & 3,09 \\
\hline Total & 7 & 100 & 2 & 100 & 13 & 100 & 43 & 100 & 22 & 100 & 28 & 100 & 44 & 100 & 14 & 100 & 21 & 100 & 194 & 100 \\
\hline \multicolumn{21}{|c|}{ Eau de puits } \\
\hline Clair & 0 & 0 & 0 & 0 & 5 & 50 & 5 & $\begin{array}{c}15,6 \\
3\end{array}$ & 0 & 0 & 0 & 0 & 6 & 54,55 & 53 & $\begin{array}{c}91,3 \\
8\end{array}$ & 4 & 50 & 73 & 55,73 \\
\hline $\begin{array}{c}\text { Troub } \\
\text { le }\end{array}$ & 7 & 100 & 0 & 0 & 5 & 50 & 27 & $\begin{array}{c}84,3 \\
8 \\
\end{array}$ & 1 & 100 & 4 & 100 & 5 & 45,45 & 5 & 8,62 & 4 & 50 & 54 & 44,27 \\
\hline Total & 7 & 100 & 0 & 0 & 10 & 100 & 32 & 100 & 1 & 100 & 4 & 100 & 11 & 100 & 58 & 100 & 8 & 100 & 131 & 100,00 \\
\hline \multicolumn{21}{|c|}{ Eau de sources naturelles } \\
\hline Clair & 4 & 100 & 1 & 100 & 0 & 0 & 1 & 100 & 3 & 100 & 1 & 100 & 6 & 100 & 31 & 100 & 0 & 0 & 47 & 100 \\
\hline Total & 4 & 100 & 1 & 100 & 0 & 0 & 1 & 100 & 3 & 100 & 1 & 100 & 6 & 100 & 31 & 100 & 0 & 0 & 47 & 100 \\
\hline
\end{tabular}

Eff. $=$ Effectif $; \%=$ Pourcentage $;$ Arr : Arrondissements

4. Evaluation de l'hygiène et de l'assainissement des ménages

4.1. Récipients de transport et de stockage

Tableau VIII. Récipient de transport de l'eau (correspond à la Figure 7a)

\begin{tabular}{|c|c|c|}
\hline Récipients de transport & Effectif & Proportion \\
\hline Bidon plastique & 620 & 42,88 \\
\hline Seau plastique & 541 & 37,41 \\
\hline Fût métallique & 11 & 0,76 \\
\hline Bidon métallique & 8 & 0,55 \\
\hline Seau métallique & 42 & 2,90 \\
\hline
\end{tabular}




\begin{tabular}{|c|c|c|}
\hline Bouteille plastique & 209 & 14,45 \\
\hline Autre & 15 & 1,04 \\
\hline Total & 1446 & 100 \\
\hline
\end{tabular}

Tableau IX. Récipient de stockage de l'eau (correspond à la Figure 7b)

\begin{tabular}{|c|c|c|}
\hline Récipients stockage & Effectif & Proportion \\
\hline Bidon plastique & 617 & 45,00 \\
\hline Seau plastique & 534 & 38,95 \\
\hline Autre & 220 & 16,05 \\
\hline Total & 1371 & 100,00 \\
\hline
\end{tabular}

Tableau X : Mode de transport

\begin{tabular}{|c|c|c|}
\hline Mode de transport & Effectif & Proportion \\
\hline Pied & 609 & 70,65 \\
\hline Brouette & 169 & 19,61 \\
\hline Voiture & 24 & 2,78 \\
\hline $\begin{array}{c}\text { Pousse-pousse } \\
\text { (charrette) }\end{array}$ & 60 & 6,96 \\
\hline Total & 862 & 100,00 \\
\hline
\end{tabular}


4.2. Durée de conservation de l'eau

Tableau XI : Durée de stockage des eaux consommées (correspond à la Figure 8)

\begin{tabular}{|c|c|c|}
\hline $\begin{array}{c}\text { Durée de } \\
\text { stockage }\end{array}$ & Effectif & Proportion \\
\hline 1 jour & 35 & 6,64 \\
\hline 2 jours & 106 & 20,11 \\
\hline 3 jours & 111 & 21,06 \\
\hline 4 jours & 61 & 11,57 \\
\hline 5 jours & 56 & 10,63 \\
\hline 6 jours & 27 & 5,12 \\
\hline 1 semaine & 63 & 11,95 \\
\hline $\begin{array}{c}\text { Plus d'une } \\
\text { semaine }\end{array}$ & 68 & 12,90 \\
\hline Total & 527 & 100,00 \\
\hline
\end{tabular}

4.3. Nettoyage et objets utilisés

Tableau XII : Fréquence de nettoyage (correspond à la Figure 9a)

\begin{tabular}{|c|c|c|}
\hline Fréquence de nettoyage & Effectif & Proportion \\
\hline Avant le remplissage & 539 & 87,64 \\
\hline $\begin{array}{c}\text { Une fois par jour ou par } \\
\text { semaine }\end{array}$ & 18 & 2,93 \\
\hline Une fois par mois & 58 & 9,43 \\
\hline Total & 615 & 100,00 \\
\hline
\end{tabular}


Tableau XIII. Objets de nettoyage (correspond à la Figure 9b)

\begin{tabular}{|c|c|c|}
\hline Objets de nettoyage & Effectif & Proportion \\
\hline Eau simple & 592 & 35,05 \\
\hline Savon & 519 & 30,73 \\
\hline Détergent (pax) & 192 & 11,37 \\
\hline Sable & 106 & 6,28 \\
\hline Détergent (Javel) & 209 & 12,37 \\
\hline Autre & 71 & 4,20 \\
\hline Total & 1689 & 100,00 \\
\hline
\end{tabular}

4.4. Utilisation et technique de traitement de l'eau à domicile

Tableau XIV. Traitement de l'eau à domicile (correspond à la Figure 10)

\begin{tabular}{|c|c|c|}
\hline Produit de traitement de l'eau & Effectif & Pourcentage \\
\hline Javel en grain & 86 & 14,55 \\
\hline Filtre moderne & 153 & 25,89 \\
\hline Eau de javel & 122 & 20,64 \\
\hline Chauffage & 168 & 28,43 \\
\hline Décantation & 62 & 10,49 \\
\hline
\end{tabular}

